Sébastien Fath, Une autre manière d'être chrétien en France. Socio-histoire de l'implantation baptiste (1810-1950)

Genève, Labor et Fides, 2001, 1222 p., (préface de Jean-Paul Willaime) (bibliogr., tablx., illustr, index) (coll. « Histoire et société », 41).

\title{
Patrick Cabanel
}

\section{(2) OpenEdition}

\section{Journals}

Édition électronique

URL : http://journals.openedition.org/assr/1227

DOI : $10.4000 /$ assr. 1227

ISSN : $1777-5825$

Éditeur

Éditions de l'EHESS

Édition imprimée

Date de publication : 1 avril 2003

Pagination : $59-157$

ISBN : 2-222-96732-5

ISSN : 0335-5985

\section{Référence électronique}

Patrick Cabanel, « Sébastien Fath, Une autre manière d'être chrétien en France. Socio-histoire de

l'implantation baptiste (1810-1950)", Archives de sciences sociales des religions [En ligne], 122 I avril - juin 2003, document 122.21, mis en ligne le 18 novembre 2005, consulté le 24 septembre 2020. URL : http://journals.openedition.org/assr/1227 ; DOI : https://doi.org/10.4000/assr.1227 
professeurs linguistes, spécialistes en langue catalane, de la même université.

Langue de culture, depuis le Moyen Âge, le Catalan est une langue romane qui n'est pas de moindre importance que les autres langues latines. Comme le français ou l'espagnol, elle est parlée ou, du moins, comprise par dix millions de personnes en Espagne. Elle est aussi coofficielle dans quatre communautés autonomes au nord et à l'est de l'Espagne: en France (Roussillon) et en Italie (nord de la Sardaigne). Les sources historiques espagnoles évoquent une traduction médiévale, à partir du latin, qui fut financée par le roi Ferdinand II d'Aragon (1479-1514) et qui reste introuvable. Plus récemment, fut éditée par l'Association Ahmadiyya, à partir de la traduction coranique anglaise, une brochure moderne, mais qui reste sélectivement limitée à quelques versets.

Cette version catalane inédite, s'affirme comme une œuvre historique grandiose sans précédent. Son intérêt ne réside pas seulement dans la publication d'une source islamique sacrée dans une langue chrétienne, mais aussi dans sa capacité à combler moult insuffisances et incompréhensions couvrant plusieurs domaines. M.D.E., a tenté d'expliquer, aux lecteurs catalans, la portée spirituelle incarnée dans le Verbe islamique sacré. À travers cette œuvre, il semble déployer beaucoup d'efforts et ménage avec délicatesse la syntaxe de la langue arabe pour essayer de préserver l'esprit sémantique $\mathrm{du}$ Texte original. Les noms propres (personnes et lieux) sont minutieusement transcrits de l'arabe puis identifiés entre parenthèses en catalan comme par exemple: (Allah/Dieu, Muhammad/Mahoma, Makka/La Meca). Les concepts sont savamment expliqués non seulement pour une meilleure compréhension du contexte, mais aussi pour mieux signifier dans un esprit d'approche les différences avec les concepts religieux monothéistes, notamment chrétiens. L'auteur et son équipe de linguistes ont réussi à trouver un style littéraire adéquat, combinant la beauté littéraire du Texte arabe, avec la disposition typographique, la ponctuation et une mélodie variée qui se rapprochent de la psalmodie du texte coranique original.

L'édition catalane du Livre Saint de l'islam, dans ces moments si particuliers, de crises marqués par la montée du fanatisme et de la désinformation, est d'une portée prodigieuse pour une compréhension réelle de l'islam à partir de sa propre source, loin de tout amalgame et perversion.

Kamel Filali.
Une autre manière d'être chrétien en France. Socio-histoire de l'implantation baptiste (1810-1950). Genève, Labor et Fides, 2001, 1222 p., (préface de Jean-Paul Willaime) (bibliogr., tablx., illustr, index) (coll. « Histoire et société », 41).

1222 pages pour 10000 personnes (dont 3000 membres baptisés) en 1950, 40000 en 2000 ? Grandeur du protestantisme, s'exclameront certains. Mais Jean-Paul Willaime a raison dans sa préface de rapprocher cet ouvrage de celui que Jean Séguy a consacré en 1977 aux assemblées anabaptistes-mennonites de France. Ce ne sont pas les chiffres qui comptent ici, mais ce que révèle sur eux et sur la société qui les entoure l'altérité de ces chrétiens, et la force de la méthode. "Une autre manière d'être chrétien »: le titre se justifie parce que l'État, d'abord, puis la société et la culture françaises, plus longuement, restent marqués par le catholicisme et ont quelque peine à accepter ou à comprendre ces protestants qui ne ressemblent pas même aux protestants "classiques", les calvinistes et les luthériens. C'est bien une autre façon de regarder et d'écrire l'histoire religieuse de la France contemporaine que S.F. propose, à l'aide d'une puissance de dépouillement, d'écriture et de réflexion peu commune. L'historien du sentiment national et du rapport à l'autre a ainsi beaucoup à puiser dans ce livre, notamment aux pages 1104-1133, où l'auteur interroge à partir du révélateur baptiste les trois paradigmes du prophète (ou du messianisme), de l'enraciné et de l'assiégé, si souvent à l'œuvre dans l'histoire des affirmations, des haines et des peurs françaises. Mais on peut aussi lire le livre comme une contribution décalée à la connaissance du protestantisme américain, ce monde qui nous reste un peu mystérieux, et dont le baptisme constitue aujourd'hui la principale dénomination, de Martin Luther King à Billy Graham (auquel S.F. vient de consacrer une étude), de Carter à Clinton.

L'historien, frotté de sociologie des religions, a organisé sa matière en trois parties : "S'établir"; "Se structurer"; "Se situer". La première partie, un livre à elle seule, d'une extrême minutie, propose l'histoire chronologique de l'implantation baptiste en France, du contexte revivaliste du début du XIX $\mathrm{X}^{\mathrm{e}}$ siècle à la stabilisation du paysage baptiste dans les années 1950. Tout est différent ici, y compris pour ceux qui connaissent l'histoire du protestantisme français : le lien avec les États-Unis (alors que les Réveils sont généralement reliés au protestantisme britannique), l'implantation prioritaire dans le nord industriel du pays, loin 
de tout foyer protestant historique, et l'origine catholique d'une majorité de pasteurs et de fidèles. On peut lire à la suite les deux derniers chapitres, portant sur une histoire politique au sens large et sur les réactions et les représentations de la population face aux baptistes. Signalons, entre autres, les pages sur les baptistes dans l'affaire Dreyfus et face au drame des Arméniens (pp. 926-933), ou leur attitude dans la crise, moins connue, de la laïcisation autoritaire à Madagascar au début du $\mathrm{XX}^{\mathrm{e}}$ siècle (pp. 919-925), ou encore sur leur action dans les années 1930 et 1940 (pp. 425-438). L'ensemble constitue une mise au point à laquelle on devra désormais se référer, y compris pour une histoire de la gestion du pluralisme religieux dans la France concordataire, peu favorable aux cultes non "reconnus".

S.F. aurait pu estimer sa tâche terminée au bout de ces 700 pages. Il a choisi, dans sa seconde partie et dans le premier chapitre de la troisième, de tenter - et de réussir - un bel essai de socio-histoire de ces chrétiens différents, dont l'organisation est caractérisée par le congrégationalisme (la “congrégation” est l'Église locale). On retiendra particulièrement le chapitre 9, sur l'identité baptiste au quotidien, avec une typologie fine : la dimension de "citadelle" (le petit troupeau des confessants résiste dans un contexte peu favorable), celle de "sentinelle" (il s'impose une fonction d'avertissement; les premiers temples baptistes ont été appelés "phares évangéliques") et celle de "passerelle" (à l'inverse de ce qui précède, le profil est plus ouvert que la tentation sectaire discernée d'abord). Qu'il s'agisse encore des réflexions sur le lien entre structure religieuse du groupe et structure politique globale, sur la dérive du leader charismatique, sur la formation de lignées baptistes (un phénomène, du reste, typique des clergés protestants ou juifs), ce livre foisonnant, parfaitement informé des classiques anciens et récents de la sociologie des religions, s'impose à l'attention.

\section{Patrick Cabanel.}

Billy Graham, pape protestant ? Paris, Albin Michel 2002, 263 p. (bibliogr., glossaire, annexes) (coll. «Sciences des religions »).

On se souvient de la surprise que créa en France, en septembre 1986, le succès de la croisade de Billy Graham à Bercy. Pourtant, la capacité d'attraction du pasteur évangéliste avait déjà été remarquée lors de ses précédentes venues en France, en 1963 à la Porte de Clignancourt, ou en 1955 au Vel d'Hiv'. Mais la portée du phénomène demeura mal appréciée, tant on connaît mal, en Europe, la place que le prédicateur protestant occupe depuis un demisiècle non seulement sur la scène religieuse américaine, mais également, à travers ses voyages incessants, sur la scène religieuse mondiale. Le grand intérêt de l'étude qu'offre S.F. sur le cas Billy Graham est de ne pas réduire la puissance de mobilisation de l'intéressé à son seul charisme personnel - au demeurant exceptionnel - pas plus qu'à sa capacité tout aussi indiscutable de maîtriser les outils de la communication de masse. Pour éclairer l'audience prodigieuse du plus célèbre "héraut de l'evangelicalism", S.F. tire plusieurs fils d'analyse dont le croisement permet de ressaisir la signification culturelle d'une trajectoire témoignant, dans son registre, d'une incomparable réussite individuelle. Le premier fil est celui du message lui-même et de son ajustement à la culture évangélique américaine. Loin de s'apparenter au type wébérien du prophète, porteur d'un message innovateur, B. Graham a en propre de tenir, à distance du fondamentalisme radical, une ligne théologique standardisée et moyenne qui fait de lui le prédicateur de référence d'une Amérique protestante à laquelle il offre, dans une fidélité bibliciste indéfectible, le discours savamment simple et efficacement rassurant de la "norme de toujours". Billy Graham, véritable homme-mémoire de l'évangélisme modéré, a fait évoluer son message - sur le terrain social par exemple, ou sur celui d'un anticatholicisme progressivement répudié - en préservant un "noyau" permanent qui assure la plausibilité de son appel constant à la conversion. S.F. montre comment cette dialectique de l'adaptation et de l'intangibilité est la clé de la dynamique consensuelle que B. Graham cristallise sur sa personne.

Le second fil de l'analyse concerne la fonctionnalité politique de la prédication grahamienne. En cette matière, deux points de vue s'opposent : celui de ceux qui mettent l'accent sur la manipulation politique à laquelle Graham, familier de tous les présidents, se serait livré tout au long de son parcours, et celui de ceux qui soulignent plutôt l'instrumentalisation dont le prédicateur a été constamment l'objet de la part des politiques. S.F. propose une autre voie d'analyse en montrant que B. Graham - ni gourou, ni pantin - pourrait bien être un "partenaire" des politiques, assurant, sur le terrain symbolique, l'expression de la religion civile américaine dont il récapitule, par la ferveur qu'il concentre autour de lui, les différentes dimensions moralisatrice, individualiste et messianique. Son intervention à la cathédrale nationale de Washington le 14 septembre 2001 a parfaitement illustré la capacité fédératrice et 